\title{
Single Silver Nanoparticles as Real-Time Optical Sensors with Zeptomole Sensitivity
}

XXXX

Vol. 0, No. 0

$\mathbf{A}-\mathbf{F}$

\author{
Adam D. McFarland and Richard P. Van Duyne* \\ Department of Chemistry, Northwestern University, 2145 Sheridan Road, \\ Evanston, Illinois 60208-3113
}

Received June 5, 2003; Revised Manuscript Received June 12, 2003

\begin{abstract}
This work utilizes dark-field optical microscopy to demonstrate the localized surface plasmon resonance $\lambda_{\text {max }}$ response of individual Ag nanoparticles to the formation of a monolayer of small-molecule adsorbates. The adsorption of fewer than 600001 -hexadecanethiol molecules on single Ag nanoparticles results in a localized surface plasmon resonance shift of $40.7 \mathrm{~nm}$. Additionally, the kinetics of the single nanoparticle response was shown to be comparable to that of other real-time sensor technologies.
\end{abstract}

Noble-metal nanoparticles have extraordinary size-dependent optical properties that have been the subject of extensive studies. ${ }^{1-4}$ Specifically, these nanoparticles exhibit a strong UV-visible extinction band that is not present in the spectrum of the bulk metal. This extinction band results when the incident photon frequency is resonant with the collective excitation of the conduction electrons and is known as the localized surface plasmon resonance (LSPR). LSPR excitation results in wavelength-selective absorption with extremely large molar extinction coefficients $\left(\sim 3 \times 10^{11} \mathrm{M}^{-1} \mathrm{~cm}^{-1}\right),{ }^{5}$ resonant Rayleigh scattering with efficiency equivalent to that of $10^{6}$ fluorophores, ${ }^{6}$ and enhanced local electromagnetic fields near the surface of the nanoparticle that are responsible for the intense signals observed in all surface-enhanced spectroscopies. ${ }^{7}$ It is well established that the peak wavelength, $\lambda_{\max }$, of the LSPR spectrum is dependent upon the size, shape, and interparticle spacing of the nanoparticle as well as its own dielectric properties and those of its local environment including the substrate, solvent, and adsorbates. ${ }^{1,8,9}$

In previous work, we have reported detailed studies of nanoparticle arrays as chemosensing and biosensing platforms. The sensing principle employed in these experiments relies on the high sensitivity of the LSPR spectrum of noblemetal nanoparticles to adsorbate-induced changes in the dielectric constant of the surrounding nanoenvironment. Arrays of size-homogeneous triangular Ag nanoparticles, fabricated using nanosphere lithography and chemically modified with a biotinylated self-assembled monolayer (SAM), were employed for the quantitative sensing of streptavidin with a subpicomolar limit of detection. ${ }^{10}$ The

\footnotetext{
* Corresponding author. E-mail: vanduyne@chem.northwestern.edu.
}

same approach has been extended for use as an immunoassay. ${ }^{11}$ The chemical modification of the nanoparticles with a SAM provides one the of keys to exploiting nanoparticle arrays as sensing platforms because the SAM can be easily tailored to incorporate a wide variety of molecular recognition elements as well as methyl- or poly(ethylene glycol)terminated alkanethiols that reduce the effect of nonspecific binding. ${ }^{10,12}$

The extension of LSPR sensing techniques to the singlenanoparticle limit provides several important improvements that will enable new nanoscale sensing experiments while retaining all of the capabilities of existing nanoparticle array and thin-film surface plasmon resonance techniques. First, the absolute detection limit (viz., number of analyte molecules per nanoparticle) is dramatically reduced. For example, the NSL-fabricated nanoparticles studied in previous work have an approximately $14000-\mathrm{nm}^{2}$ surface area accessible to molecular adsorbates. ${ }^{13}$ Because the packing density of 1-hexadecanthiol (1-HDT) is 4.4 molecules $\mathrm{nm}^{-2}$, the maximum number of 1-HDT molecules adsorbed to each nanoparticle is $\sim 60000$ ( $\sim 100$ zeptomoles $)$. The formation of a 1-HDT monolayer on the nanoparticle array results in an LSPR $\lambda_{\max }$ shift of greater that $40 \mathrm{~nm}$, a change that is approximately 100 times larger than the instrumental resolution of typical small-footprint UV-visible spectrophotometers. This suggests that the limit of detection for singlenanoparticle-based LSPR sensing is well below 1000 molecules for small-molecule adsorbates. For larger molecules, such as antibodies and proteins that result in a greater change in the local dielectric environment per adsorbed molecule, detection limits are even further improved. Riboh et al. determined that antibiotin occupies approximately 150 
$\mathrm{nm}^{2}$ molecule ${ }^{-1}$ when bound to NSL-fabricated particles that were modified with a biotinylated-SAM. ${ }^{11}$ The saturation response of the LSPR shift, which corresponds to the detection of fewer than 100 molecules nanoparticle ${ }^{-1}$, was $38 \mathrm{~nm}$. This suggests that the sensitivity of single-nanoparticle LSPR spectroscopy could approach the single-molecule limit of detection for large biomolecules. Additionally, this extreme sensitivity dictates that only very small sample volumes (viz., attoliters) are necessary to achieve a measurable response.

A second advantage of utilizing single nanoparticles as sensors lies in their noninvasive nature, making them ideal platforms for in vivo quantification of chemical species and monitoring of dynamic processes inside biological cells. The development of methods to incorporate single-nanoparticle sensors into organs, tissue, and cells is facilitated by the fact that chemical modification using a SAM is a straightforward means of increasing the biocompatibility of nanoparticles. ${ }^{14,15}$ Kneipp et al. have explored the incorporation of $\mathrm{Ag}$ nanoparticles into living cells in order to probe the cell contents via surface-enhanced Raman spectroscopy. ${ }^{16}$ Furthermore, $\mathrm{Xu}$ et al. have monitored the transmembrane transport dynamics of Au nanoparticles using dark-field LSPR spectroscopy. ${ }^{17}$

Single-nanoparticle sensing platforms offer further advantages because they are readily implemented in multiplex detection schemes. By controlling the size, shape, and chemical modification of individual nanoparticles, several sensing platforms can be fabricated in which each unique nanoparticle can be distinguished from others on the basis of the spectral location of its LSPR $\lambda_{\max }$. Several of these unique nanoparticles can then be incorporated into one device, allowing for the rapid, simultaneous, label-free detection of thousands of different chemical or biological species. For example, if each nanoparticle is spaced by 2 to 3 times the Rayleigh limit from adjacent nanoparticles, a 50 $\times 50 \mu \mathrm{m}^{2}$ array could be used to perform simultaneous screening for as many as 2500 different analytes.

Another key to exploiting single nanoparticles as sensing platforms lies in the development of a technique to measure the LSPR spectrum of individual nanoparticles with a high signal-to-noise ratio. UV-visible extinction spectroscopy does not provide a practical means of accomplishing this task. Even under the most favorable experimental conditions, the absorbance of a single nanoparticle is very close to the shot noise-governed detection limit. Instead, resonant Rayleigh scattering spectroscopy is the most straightforward means of characterizing the LSPR spectrum of individual noble-metal nanoparticles. In a manner similar to fluorescence spectroscopy, the advantage of scattering spectroscopy lies in the fact that the scattering signal is being detected in the presence of a very low background. The instrumental approach for performing these experiments generally involves using high-magnification microscopy coupled with the oblique or evanescent illumination of the nanoparticles. Klar et al. utilized a near-field scanning optical microscope coupled to a tunable laser source to measure the scattering spectra of individual gold nanoparticles embedded in a $\mathrm{TiO}_{2}$ film. ${ }^{18}$ Sonnichsen et al. measured the scattering spectra of individual nanoparticles that were fabricated using electron beam lithography. ${ }^{19}$ Their technique involved illuminating the nanoparticles with the evanescent field produced by the total internal reflection of light in a glass prism. The light scattered by the nanoparticles was collected with a microscope objective and coupled into a spectrometer for analysis. Matsuo and Sasaki employed differential interference contrast microscopy to perform time-resolved laser scattering spectroscopy of single silver nanoparticles..$^{20}$ Mock et al. correlated conventional dark-field microscopy and TEM to investigate the relationship between the structure of individual metallic nanoparticles and their scattering spectra. ${ }^{21}$ They have also used the same light microscopy techniques to study the response of the scattering spectrum to the nanoparticle's local dielectric environment by immersing the nanoparticle in oils of various refractive indexes..$^{22}$ Although previous studies have optically characterized individual noble-metal nanoparticles in various dielectric environments, no studies have been done to investigate the sensitivity of single $\mathrm{Ag}$ nanoparticles to the presence of molecular adsorbates. The work presented here utilizes dark-field microscopy to demonstrate the LSPR $\lambda_{\max }$ response of individual Ag nanoparticles to the formation of a monolayer of small-molecule adsorbates. ${ }^{23}$

Chemically synthesized Ag nanoparticles were prepared by reducing silver nitrate with sodium citrate in aqueous solution according to the procedure described by Lee and Miesel. ${ }^{24}$ The majority of the nanoparticles prepared using this technique are spherical with an average diameter of 35 $\mathrm{nm}$, but prisms, platelets, disks, and rods of various sizes are also present. The nanoparticles were immobilized on a no. 1 coverslip by drop coating approximately $5 \mu \mathrm{L}$ of the colloidal solution onto the substrate and allowing the water to evaporate. The coverslip was then inserted into a customdesigned flow cell. Prior to all experiments, the nanoparticles in the flow cell were rinsed repeatedly with methanol and dried under nitrogen to establish an equilibrium surface adsorption of solvent molecules and citrate anions. All optical measurements were performed using an inverted microscope (Eclipse TE300, Nikon Instruments) equipped with an imaging spectrograph (SpectroPro 300i, Roper Scientific) and a CCD detector (Spec-10:400B, Roper Scientific). A color video camera was also attached to the front port. A darkfield condenser $(\mathrm{NA}=0.95)$ was used to illuminate the nanoparticles and a variable-aperture $100 \times$ oil-immersion objective (NA $=0.5-1.3$ ) was used to collect the light scattered by the nanoparticles. Figure 1 shows an image of a field of $\mathrm{Ag}$ nanoparticles acquired with the experimental apparatus.

The local refractive index sensitivity of the LSPR spectrum of a single Ag nanoparticle was measured by recording the resonant Rayleigh scattering spectrum of the nanoparticle as it was exposed to various solvent environments inside the flow cell. As illustrated in Figure 2, the LSPR $\lambda_{\max }$ systematically shifts to longer wavelength as the solvent refractive index is increased. Linear regression analysis for this nanoparticle yielded a refractive index sensitivity of 


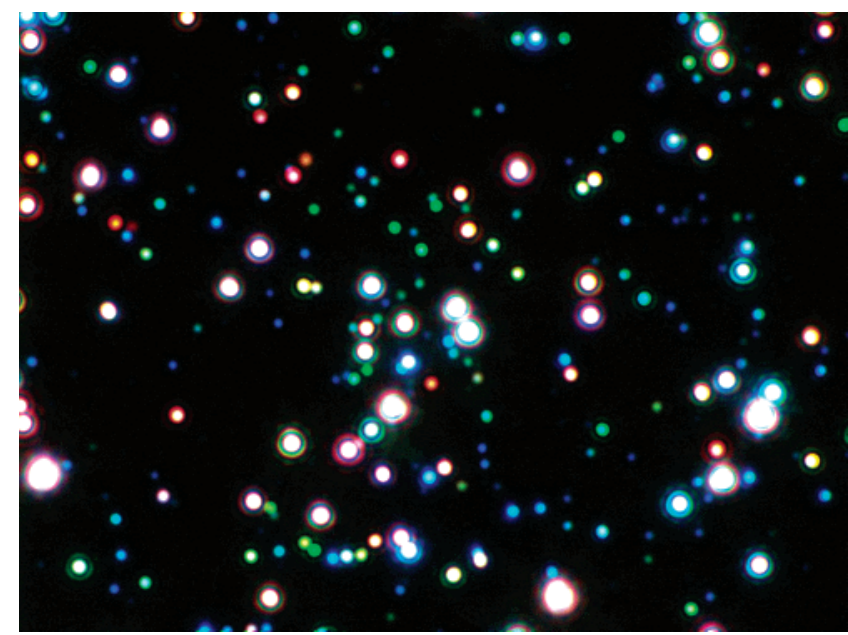

Figure 1. Dark-field optical image of Ag nanoparticles. The field of view is approximately $130 \times 170 \mu \mathrm{m}^{2}$.

$203.1 \mathrm{~nm} \mathrm{RIU}{ }^{-1}$. The refractive index sensitivity of several individual chemically synthesized $\mathrm{Ag}$ nanoparticles was measured, and typical values were determined to be $160-$ $235 \mathrm{~nm} \mathrm{RIU}^{-1}$.

The sensitivity of the LSPR $\lambda_{\max }$ to changes in the local dielectric environment is determined by differences in nanoparticle geometry. According to Mie theory, the extinction of a single spherical metal nanoparticle is governed by the following equation: $:^{1,25,26}$

$$
E(\lambda)=\frac{24 \pi^{2} a^{3} \epsilon_{\mathrm{m}}^{3 / 2}}{\lambda \ln (10)}\left[\frac{\epsilon_{\mathrm{i}}(\lambda)}{\left(\epsilon_{\mathrm{r}}(\lambda)+2 \epsilon_{\mathrm{m}}\right)^{2}+\epsilon_{\mathrm{i}}(\lambda)^{2}}\right]
$$

$E(\lambda)$ is the extinction (viz., sum of absorption and scattering) magnitude, $a$ is the radius of the metallic nanosphere, $\epsilon_{\mathrm{m}}$ is the dielectric constant of the medium surrounding the metallic

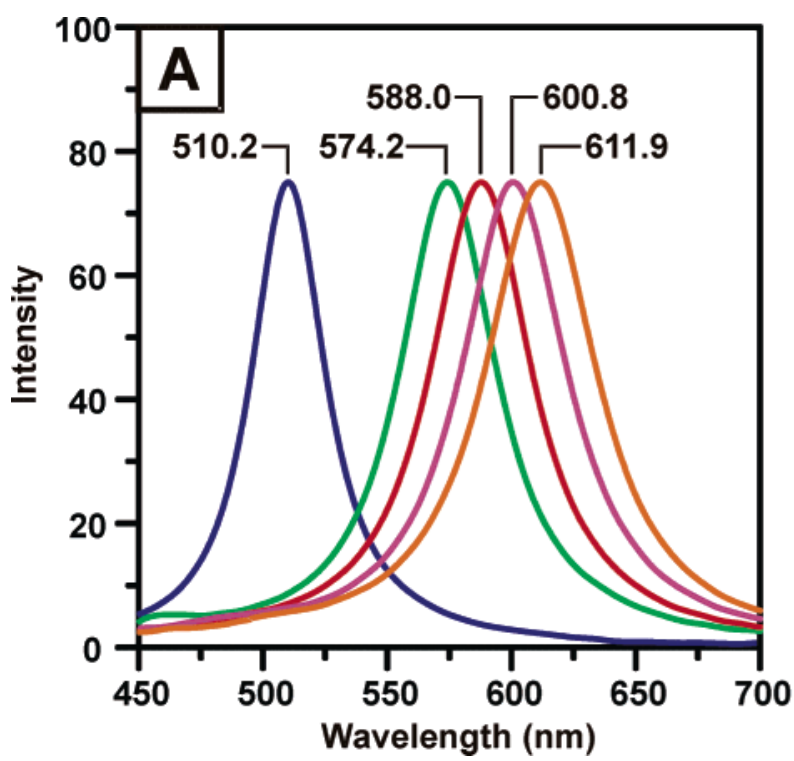

nanosphere $\left(\epsilon_{\mathrm{m}}\right.$ is assumed to be a positive, wavelengthindependent, real number), $\lambda$ is the wavelength of the incident radiation, $\epsilon_{\mathrm{i}}(\lambda)$ is the imaginary portion of the metallic nanosphere's wavelength-dependent dielectric function, and $\epsilon_{\mathrm{r}}(\lambda)$ is the real portion of the metallic nanosphere's wavelength-dependent dielectric function. The LSPR $\lambda_{\max }$ occurs when the denominator of the term in brackets approaches zero. This occurs at the wavelength where $\epsilon_{\mathrm{i}}(\lambda)$ is small and $\epsilon_{\mathrm{r}}(\lambda)=-2 \epsilon_{\mathrm{m}}$. For nonspherical nanoparticles, eq 1 is modified such that the LSPR $\lambda_{\max }$ occurs when $\epsilon_{\mathrm{r}}(\lambda)$ $=-\chi \epsilon_{\mathrm{m}}$. The factor $\chi$ accounts for nanoparticle shape and is a function of the nanoparticle aspect ratio. For a given change in the local dielectric environment $\left(\epsilon_{\mathrm{m}}\right), \chi$ dictates the sensitivity of the LSPR $\lambda_{\max }$ response (i.e., a larger $\chi$ results in a greater refractive index sensitivity). For spherical nanoparticles, $\chi=2$, but $\chi$ increases as the aspect ratio of the nanoparticle increases. For example, $\chi \approx 16$ for an ellipsoidal nanoparticle with an aspect ratio of 5:1, which translates to a dielectric sensitivity that is 5-10 times greater than that for a spherical nanoparticle. A detailed description of the relationship between $\chi$ and ellipsoidal nanoparticle structure has been documented elsewhere. ${ }^{8}$

To illustrate the effect of nanoparticle shape on LSPR $\lambda_{\max }$ dielectric sensitivity, the LSPR spectra of three differently shaped nanoparticles were collected in various solvent environments (Figure 3). The triangular nanoparticle was fabricated using NSL, removed from its original substrate, and immobilized on a glass coverslip to ensure adequate interparticle separation. The geometries of the chemically synthesized nanoparticles were assigned on the basis of the LSPR $\lambda_{\max }$, line shape, and polarization dependence of the scattering spectra as well as similar assignments made by Mock et al. using TEM-correlated optical measurements. ${ }^{21}$ The narrow width and symmetric appearance of the LSPR peaks also indicate that individual nanoparticles, as opposed to small aggregates, are being examined. As predicted by

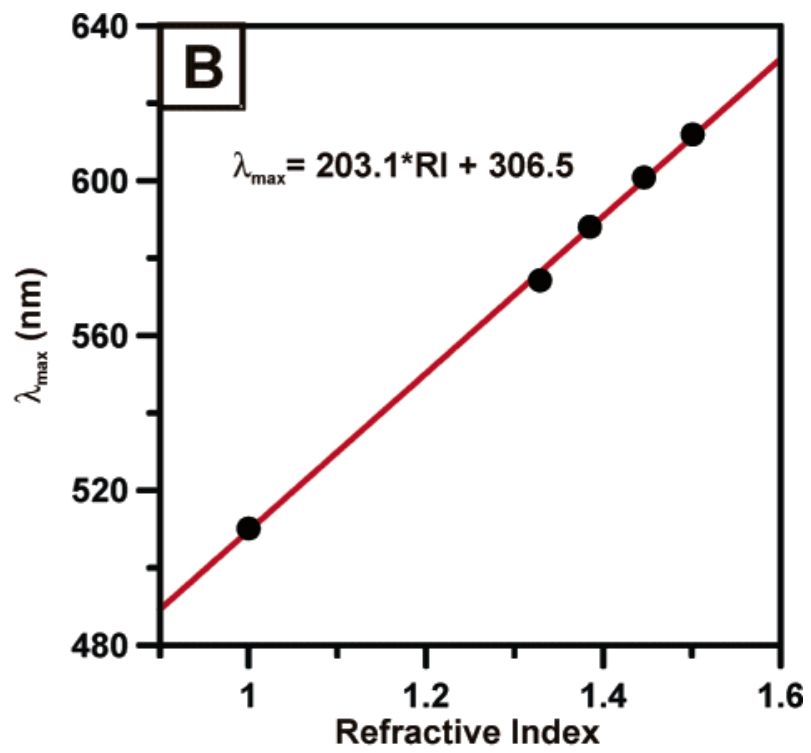

Figure 2. (A) Single Ag nanoparticle resonant Rayleigh scattering spectrum in various solvent environments (left to right): nitrogen, methanol, 1-propanol, chloroform, and benzene. (B) Plot depicting the linear relationship between the solvent refractive index and the LSPR $\lambda_{\max }$. 


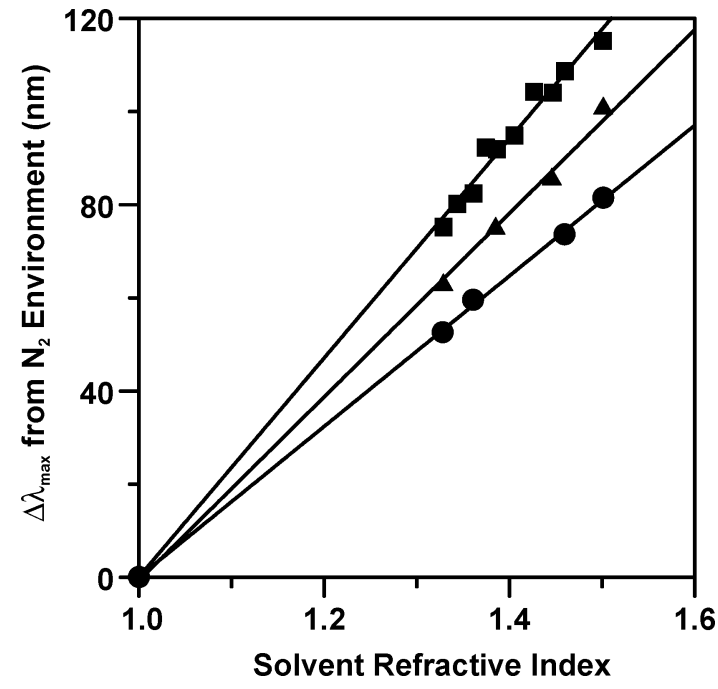

Figure 3. Comparison of refractive index sensitivity for $\mathrm{Ag}$ nanoparticles with different geometries. The spherical nanoparticle (O) has a sensitivity of $161 \mathrm{~nm} \mathrm{RIU}{ }^{-1}$, the triangular nanoparticle (A) has a sensitivity of $197 \mathrm{~nm} \mathrm{RIU}{ }^{-1}$, and the rodlike nanoparticle (ם) has a sensitivity of $235 \mathrm{~nm} \mathrm{RIU}{ }^{-1}$.

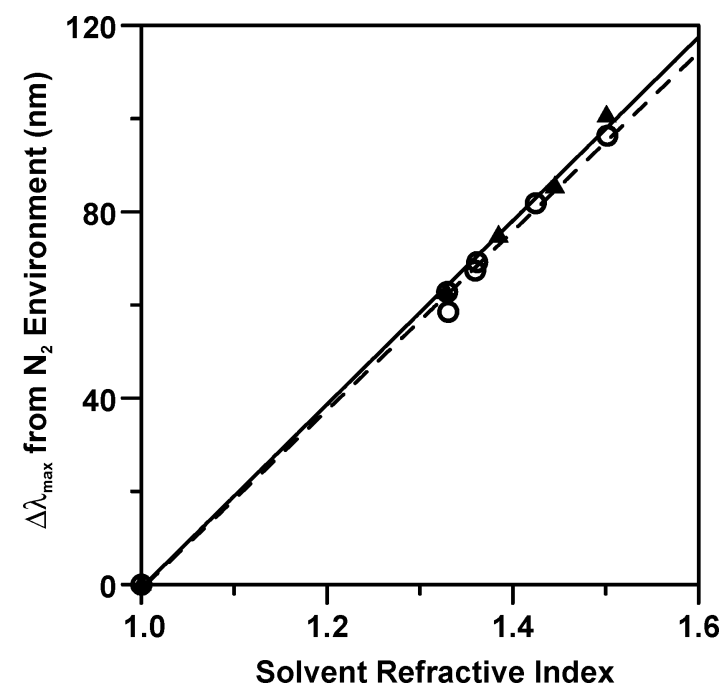

Figure 4. Comparison of the refractive index sensitivity of an individual $\mathrm{Ag}$ triangular nanoparticle $(\boldsymbol{\Delta})$ to an NSL-fabricated nanoparticle array (O). The respective sensitivities are 197 and 191 $\mathrm{nm} \mathrm{RIU}^{-1}$.

the theory outlined above, the nanoparticles with larger aspect ratios exhibit higher sensitivity to changes in the local dielectric environment. In the case of the triangular nanoparticle, the dielectric sensitivity is very similar to values reported for NSL-fabricated nanoparticle arrays (Figure 4). ${ }^{13}$ This suggests that all of the chemosensing and biosensing techniques that have been developed using NSL-fabricated arrays can be directly translated into a single nanoparticle format.

To investigate the application of single nanoparticles as chemical-sensing platforms, the LSPR $\lambda_{\max }$ response to molecular adsorbates was probed. After recording the resonant Rayleigh scattering spectrum of an individual $\mathrm{Ag}$ nanoparticle in a nitrogen environment, a $1.0 \mathrm{mM}$ alkanethiol solution in ethanol was injected into the flow cell. The

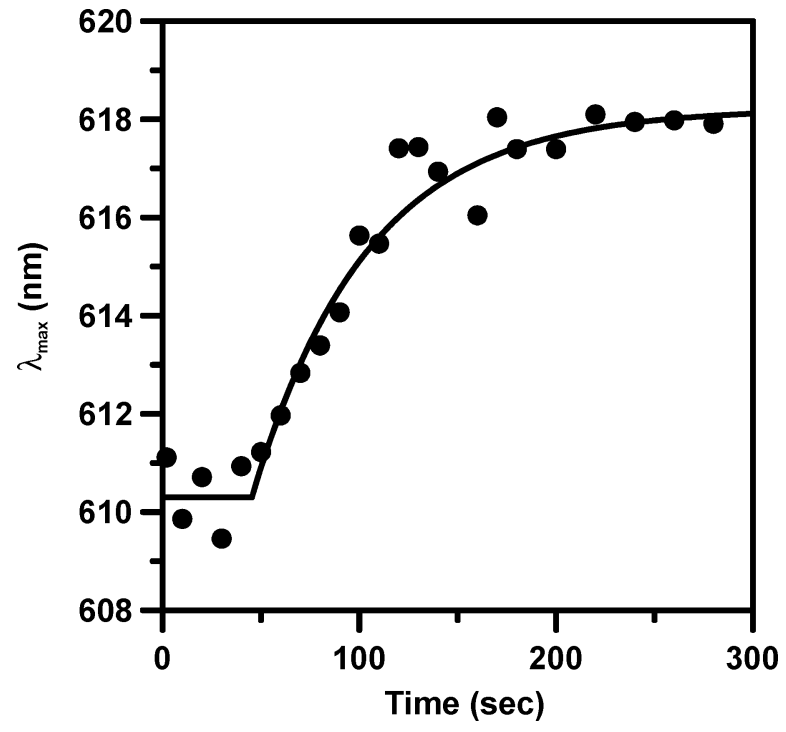

Figure 5. Real-time LSPR response of a single Ag nanoparticle as $1.0 \mathrm{mM} 1$-octanethiol was injected into the flow cell. The circles represent the experimental data, and the line is a first-order response profile with a rate constant of $0.0167 \mathrm{~s}^{-1}$.

nanoparticles were incubated in the solution for at least $1 \mathrm{~h}$, even though real-time monitoring of the scattering spectrum indicates that the majority of the response occurs in the first few minutes after injection. Figure 5 illustrates the real-time LSPR $\lambda_{\max }$ response of a nanoparticle exposed to $1.0 \mathrm{mM}$ 1 -octanethiol in ethanol. An analysis of the data reveals that the response exhibits first-order kinetics with a rate constant of $0.0167 \mathrm{~s}^{-1}$. This kinetic response demonstrates that singlenanoparticle-sensing platforms provide the same advantageous real-time behavior observed in nanoparticle array ${ }^{11}$ and thin-film surface plasmon resonance ${ }^{12,27,28}$ sensor technologies.

After incubation in the analyte solution, the flow cell was flushed several times with ethanol, methanol, and hexane to ensure that no more than one monolayer of adsorbate had accumulated on the surface. Finally, the nanoparticle was then dried under nitrogen, and a scattering spectrum was collected. Figure 6A displays the scattering spectrum of an individual $\mathrm{Ag}$ nanoparticle in nitrogen before and after modification with 1-HDT. The 1-HDT SAM formation resulted in a 40.7-nm red shift in the LSPR $\lambda_{\max }$. On the basis of a conservative estimate of the maximum possible surface area of the nanoparticle examined and the monolayer packing density of 1-HDT on Ag, this response corresponds to the detection of fewer than 60000 adsorbate molecules.

The LSPR $\lambda_{\max }$ response to various SAM chain lengths was measured using different length alkanethiols. In an effort to ensure that $\mathrm{Ag}$ nanoparticles of similar size and shape were used in this study, only nanoparticles exhibiting similar LSPR $\lambda_{\max }$ and refractive index sensitivities were used. The LSPR $\lambda_{\max }$ (in a nitrogen environment) of the nanoparticles that were studied occurred in the range of $510-522 \mathrm{~nm}$, and their refractive index sensitivities ranged from 193 to 204 $\mathrm{nm} \mathrm{RIU}{ }^{-1}$. These spectral similarities ensured that consistent nanoparticle geometry was used during consecutive experiments. An analysis of the scattering spectra of these 

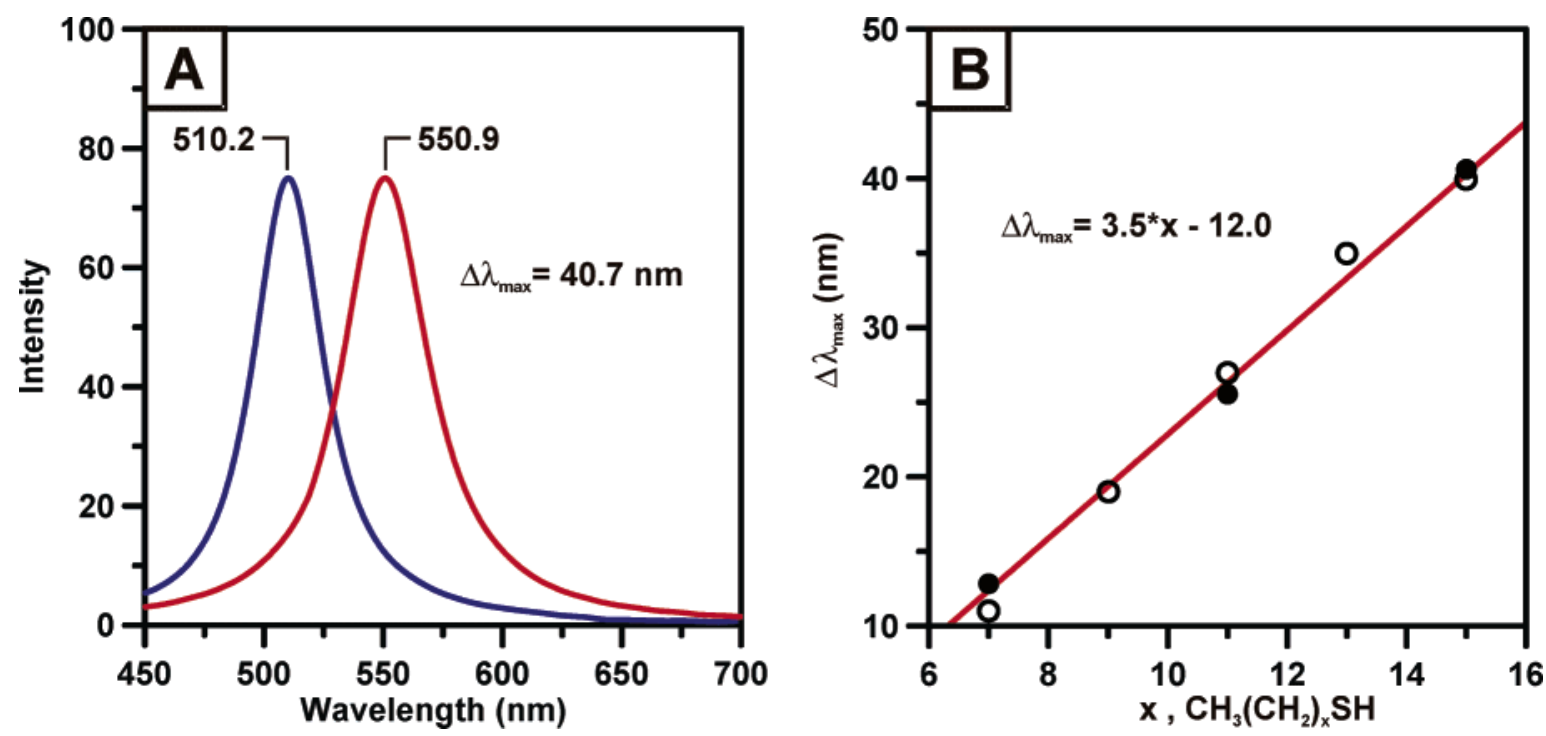

Figure 6. (A) Individual Ag nanoparticle before and after modification with 1-HDT. (B) Plot depicting the linear relationship between the LSPR response and the SAM alkyl chain length. The solid circles and linear regression are for the single-nanoparticle experiments, and the open circles are the data points for the NSL-fabricated nanoparticle array experiments.

nanoparticles suggests that they are most likely platelets or disks. Figure 6B shows the single-nanoparticle LSPR $\lambda_{\max }$ response for the formation of three SAMs of varied alkyl chain length. The observed response is linear with a sensitivity of $+3.5 \mathrm{~nm}$ (positive values signify a shift to longer wavelengths) for each additional methylene unit in the alkanethiol that comprises the SAM. The ordinate intercept of the linear fit is $-12.0 \mathrm{~nm}$. This calculated blue shift is attributed to the electron-donating nature of the sulfur headgroup of the molecular adsorbates, which shifts the LSPR $\lambda_{\max }$ to shorter wavelengths. Once again, the magnitudes of the response are very similar to those of previous measurements of the formation of alkanethiol SAMs on NSLfabricated nanoparticle arrays as shown in Figure 6B. ${ }^{13}$ Detailed analyses of the LSPR $\lambda_{\max }$ response to dielectric overlayers of various thicknesses can be found elsewhere. ${ }^{11,13,27}$ Mie theory calculations performed on spherical nanoparticles predict the linear behavior of the response shown in Figure 6B but underestimate the sensitivity. ${ }^{13}$ The higher sensitivity observed in our past and present work is due to the nanoparticles exhibiting $\chi$ values greater than 2 .

In summary, this work demonstrates the first measurement of the LSPR $\lambda_{\max }$ response to the formation of a SAM on single Ag nanoparticles. The spectral shifts observed for these systems correspond to sensing fewer than 60000 smallmolecule adsorbates. Additionally, the kinetics of the singlenanoparticle LSPR $\lambda_{\max }$ response was shown to be comparable to the kinetics of other real-time sensor technologies. This represents a significant step toward the implementation of individual $\mathrm{Ag}$ nanoparticles as quantitative real-time biosensing platforms. We anticipate that future development of single-nanoparticle-sensing platforms will (1) approach real-time sensitivities of a few molecules or perhaps even that of a single molecule; (2) allow massively parallel bioassays and immunoassays; and (3) facilitate the development of noninvasive in vivo sensing techniques for the study of biological samples.
Acknowledgment. We acknowledge the support of the Nanoscale Science and Engineering Initiative of the National Science Foundation under NSF award number EEC-0118025. Any opinions, findings, and conclusions or recommendations expressed in this material are those of the authors and do not necessarily reflect those of the National Science Foundation. We also acknowledge the support of the Air Force Office of Scientific Research Multidisciplinary University Research Initiative program (F49620-02-1-0381) and the ACS Petroleum Research Fund (PRF 37982-AC5). We are grateful for useful discussion and technical support provided by Professor G. C. Schatz and Dr. C. L. Haynes.

Note Added in Proof. After submission of this manuscript, the existence of another paper containing material related to this work was brought to our attention. ${ }^{29}$

\section{References}

(1) Haynes, C. L.; Van Duyne, R. P. J. Phys. Chem. B 2001, 105, 55995611.

(2) El-Sayed, M. A. Acc. Chem. Res. 2001, 34, 257-264.

(3) Kneipp, K.; Kneipp, H.; Itzkan, I.; Dasari, R. R.; Feld, M. S. Chem Rev. 1999, 99, 2957-2975.

(4) Kreibig, U. In Handbook of Optical Properties: Optics of Small Particles, Interfaces, and Surfaces; Hummel, R. E., Wissmann, P., Eds.; CRC Press: Boca Raton, FL, 1997; Vol. 2, pp 145-190.

(5) Jensen, T. R.; Duval Malinsky, M.; Haynes, C. L.; Van Duyne, R. P. J. Phys. Chem. B 2000, 104, 10549-10556.

(6) Yguerabide, J.; Yguerabide, E. E. Anal. Biochem. 1998, 262, 157176.

(7) Schatz, G. C.; Van Duyne, R. P. In Handbook of Vibrational Spectroscopy; Chalmers, J. M., Griffiths, P. R., Eds.; Wiley: New York, 2002; Vol. 1, pp 759-774.

(8) Kelly, K. L.; Coronado, E.; Zhao, L.; Schatz, G. C. J. Phys. Chem. B 2003, 107, 668-677.

(9) Haynes, C. L.; McFarland, A. D.; Zhao, L.; Van Duyne, R. P.; Schatz, G. C.; Gunnarsson, L.; Prikulis, J.; Kasemo, B.; Käll, M. J. Phys. Chem. B, in press, 2003.

(10) Haes, A. J.; Van Duyne, R. P. J. Am. Chem. Soc. 2002, 124, 1059610604.

(11) Riboh, J. C.; Haes, A. J.; McFarland, A. D.; Ranjit, C.; Van Duyne, R. P. J. Phys. Chem. B 2003, 107, 1772-1780.

(12) Mrksich, M.; Grunwell, J. R.; Whitesides, G. M. J. Am. Chem. Soc. 1995, 117, 12009-12010. 
(13) Malinsky, M. D.; Kelly, K. L.; Schatz, G. C.; Van Duyne, R. P. J. Am. Chem. Soc. 2001, 123, 1471-1482.

(14) Schneider, T. W.; Aloi, L. E.; White, R. C. SPIE Proc. 2000, 4205, 128-134.

(15) Bell, D. J.; Brody, J. P.; Yager, P. SPIE Proc. 1998, 3258, 134139.

(16) Kneipp, K.; Haka, A. S.; Kneipp, H.; Badizadegan, K.; Yoshizawa, N.; Boone, C.; Shafer-Peltier, K. E.; Motz, J. T.; Dasari, R. R.; Feld, M. S. Appl. Spectrosc. 2002, 56, 150-154.

(17) Xu, X.-H. N.; Chen, J.; Jeffers, R. B.; Kyriacou, S. Nano Lett. 2002, $2,175-182$.

(18) Klar, T.; Perner, M.; Grosse, S.; von Plessen, G.; Spirkl, W.; Feldmann, J. Phys. Rev. Lett. 1998, 80, 4249-4252.

(19) Sönnichsen, C.; Geier, S.; Hecker, N. E.; von Plessen, G.; Feldmann, J.; Ditlbacher, H.; Lamprecht, B.; Krenn, J. R.; Aussenegg, F. R.; Chan, V. Z.-H.; Spatz, J. P.; Möller, M. Appl. Phys. Lett. 2000, 77, 2949-2951.

(20) Matsuo, Y.; Sasaki, K. Jpn. J. Appl. Phys. 2001, 40, 61436147.
(21) Mock, J. J.; Barbic, M.; Smith, D. R.; Schultz, D. A.; Schultz, S. J. Chem. Phys. 2002, 116, 6755-6759.

(22) Mock, J. J.; Smith, D. R.; Schultz, S. Nano Lett. 2003, 3, 485491.

(23) Van Duyne, R. P.; McFarland, A. D. Nanoparticulate Sensors. U.S Provisional Patent Application NU 22078/23008, February 20, 2003.

(24) Lee, P. C.; Meisel, D. J. Phys. Chem. 1982, 86, 3391-3395.

(25) Link, S.; El-Sayed, M. A. J. Phys. Chem. B 1999, 103, 84108426.

(26) Kreibig, U.; Vollmer, M. Optical Properties of Metal Clusters; Springer-Verlag: Heidelberg, Germany, 1995; Vol. 25.

(27) Jung, L. S.; Campbell, C. T.; Chinowsky, T. M.; Mar, M. N.; Yee, S. S. Langmuir 1998, 14, 5636-5648.

(28) Peterlinz, K. A.; Georgiadis, R. Langmuir 1996, 12, 4731-4740.

(29) Raschke, G.; Kowarik, S.; Franzl, T.; Sönnichsen, C.; Klar, T. A.; Feldmann, J.; Nichtl, A.; Kürzinger, K. Nano Lett. 2003, in press. NL034372S 